\title{
Die Pandemie hat uns wieder im Würgegriff
}

\section{Daniel Schlossberg}

Dr. med., Facharzt für Allgemeine Innere Medizin, Mitglied FMH, Zürich

\section{Die Menschen wissen, was immer sie gegen die neuen Coronaviren unternehmen, es müsste durchgreifend sein. Und das wird zunehmend schwierig. Die Wider- stände in der Gesellschaft haben zugenommen.}

Nach einer kurzen Verschnaufpause im Sommer haben sie ihre Kräfte gesammelt. An einer Sitzung der Stammesführer ergreift das Oberhaupt der D614GMutante, genannt der Fade, das Wort. Er führt ganz unscheinbar den Stamm an, der sich bei uns am raschesten verbreitet. «Wir haben die Schwäche der Menschen ausgenützt, die im Sommer getanzt, gesungen und gefeiert haben. Und die unsere Existenz leugnen.» Unbemerkt haben sie sich ihren Weg gebahnt, die Jungen befallen, die es oft gar nicht bemerkt haben. Jene haben sie weitergereicht und nun wieder zurück in ältere Populationen getragen. Da fordern sie ihre Opfer. Die

Das hilft denen, die wieder da sind, da, um zu bleiben und weiter anzustecken.

Menschen haben gesagt, sie wüssten nicht, warum die Fallzahlen wieder so rasch angestiegen seien. Den neuen Coronaviren kann das nur recht sein. Solange sie es nicht wüssten, könnten sie ungestört ihr Werk fortführen. Der Fade und der Wilde, Anführer des Wildtyps, sind sich einig, dass sie an Schlagkraft gewonnen hätten durch die raschere Vermehrung in ihren Wirten, ohne diese kränker zu machen. Nur so hätten sie ihren Siegeszug fortsetzen können. Sie hätten schliesslich von ihren Artgenossen gelernt, die die Leute zu Beginn des Jahrtausends zu krank gemacht und sich so selber ausgelöscht hätten. Sie seien auf Gedeih und Verderb auf ihre Gastwirte angewiesen. Sie sind wieder da, und wie! Die Menschen konstatierten im Oktober, es sei fünf vor zwölf und mithin gut, dass die Uhren mit Beginn der Winterzeit um eine Stunde zurückgedreht würden. Doch sie wissen, dass es nichts ändert und die Sache nun ihren Lauf nimmt. Das sei das Präventionsparadoxon. Wenn Vorbeugen noch heilen würde, würden sie nichts davon wissen wollen, weil sie nichts merken. Sterben gehöre zum Leben, dieses Schicksal sei allen beschieden, die Frage sei bloss wann. Allmählich kenne doch jeder einen, der es mit ihnen zu tun gehabt habe. Nicht immer sei es glimpflich verlaufen. Doch weil die Menschen sich nicht erinnern, was sie vor einer Woche gemacht haben, bleibt die Quelle eines Ausbruchs meistens unentdeckt. Das hilft denen, die wieder da sind, da, um zu bleiben und weiter anzustecken. Einmal da, sind sie kaum zu vertreiben. Sie nützen die Versäumnisse der Fahnder, die auf das Ziel statt auf die Quelle fokussieren. So kommen sie nie an die Orte hin, wo das Infektionsgeschehen vorangetrieben wird, und hinken diesem hoffnungslos hinterher. So geht das! Jenen Faden und Wilden kann das nur recht sein, zumal es den Mutanten in Nerzen nun an den Kragen gegangen ist.

Die Politiker haben sich versammelt und beschliessen Massnahmen. Sie wollen noch mehr testen, aber sie weisen asymptomatische Kontakte von Infizierten weiterhin ab. Sie wollen nun doch die Quellcluster suchen, doch bei tausenden von Neuinfizierten jeden Tag ist das Backward-Tracing ein hoffnungsloses Unterfangen. So hoffen die, die wieder da sind, hier bleiben zu können, weil jene, die sie vertreiben könnten, nicht zu wirksamen Massnahmen greifen. Wenigstens vorübergehend. Denn Impfungen sind unterwegs. Doch zum Glück gibt es da noch die Impfskepsis der Menschen. Das hilft jenen, die wieder da sind, dazubleiben. Schon mit dem Maskentragen tun sich die Menschen schwer. Die Brillengläser laufen an, auf Smartphones funktioniert die Gesichtserkennung nicht. Würden Sie es dennoch konsequent tun, liesse sich die Seuche wirksam eindämmen. Die Faden und die Wilden kümmert es nicht, sie sind einfach da. Die meisten Menschen hätten im Sommer gehofft, dass die Coronaviren sterben würden, doch sie sind - mehr denn je - wieder da!

Bildnachweis

(c) Katerynakon | Dreamstime.com, Symbolbild 